\title{
GEOMETRIC POINT QUALITY ASSESSMENT FOR THE AUTOMATED, MARKERLESS AND ROBUST REGISTRATION OF UNORDERED TLS POINT CLOUDS
}

\author{
M. Weinmann, B. Jutzi
}

Institute of Photogrammetry and Remote Sensing, Karlsruhe Institute of Technology (KIT)

Englerstr. 7, 76131 Karlsruhe, Germany - \{martin.weinmann, boris.jutzi\}@kit.edu

Commission III, WG III/2

KEY WORDS: Laser scanning, TLS, point cloud, imagery, filtering, feature extraction, matching, registration

\begin{abstract}
:
The faithful 3D reconstruction of urban environments is an important prerequisite for tasks such as city modeling, scene interpretation or urban accessibility analysis. Typically, a dense and accurate 3D reconstruction is acquired with terrestrial laser scanning (TLS) systems by capturing several scans from different locations, and the respective point clouds have to be aligned correctly in a common coordinate frame. In this paper, we present an accurate and robust method for a keypoint-based registration of unordered point clouds via projective scan matching. Thereby, we involve a consistency check which removes unreliable feature correspondences and thus increases the ratio of inlier correspondences which, in turn, leads to a faster convergence of the RANSAC algorithm towards a suitable solution. This consistency check is fully generic and it not only favors geometrically smooth object surfaces, but also those object surfaces with a reasonable incidence angle. We demonstrate the performance of the proposed methodology on a standard TLS benchmark dataset and show that a highly accurate and robust registration may be achieved in a fully automatic manner without using artificial markers.
\end{abstract}

\section{INTRODUCTION}

The faithful 3D reconstruction of urban environments represents a topic of great interest in photogrammetry, remote sensing and computer vision, as it provides an important prerequisite for applications such as city modeling, scene interpretation or urban accessibility analysis. While a variety of devices allows for acquiring an appropriate representation of object surfaces in the form of 3D point cloud data, terrestrial laser scanning (TLS) systems provide dense and accurate point cloud data for the local environment and they may also reliably measure distances of several tens of meters. However, a TLS system is a line-of-sight instrument and hence occlusions resulting from objects in the scene may be expected as well as a significant variation in point density between close and distant object surfaces. Consequently, multiple point clouds have to be acquired from different locations in order to obtain complete objects and full scene coverage. As the spatial 3D coordinates of each of these point clouds are only determined w.r.t. the local coordinate frame of the sensor, all captured point cloud data have to be transferred into a common coordinate frame. This process is commonly referred to as point cloud registration, point set registration or 3D scan matching.

In this paper, we focus on keypoint-based point cloud registration which has proven to be among the most efficient strategies for aligning pairs of overlapping scans. Generally, such keypointbased point cloud registration approaches rely on $(i)$ forwardprojected 2D keypoints detected in either intensity or range images, or (ii) 3D keypoints extracted from either the original point cloud data or a voxel-based subsampling in order to obtain an approximately homogeneous point density. For reasons of efficiency and robustness, we exploit forward-projected 2D keypoints and thereby take into account that not all of the detected correspondences between different scans are guaranteed to contain reliable 3D information. We explicitly address the latter issue by presenting a new measure for point quality assessment. This measure is fully generic and it not only favors geometrically smooth object surfaces, but also those object surfaces with a reasonable incidence angle which, in turn, efficiently handles unreliable range measurements arising from large incidence angles.
In summary, we $(i)$ present a new measure for assessing the quality of scanned 3D points in a fully generic manner, (ii) explain the pros and cons of this measure in comparison to other alternatives, (iii) define a consistency check based on the new measure for filtering unreliable feature correspondences and (iv) demonstrate the significance of the consistency check for an efficient and robust registration of TLS point clouds.

After reflecting related work on point quality assessment, feature extraction and point cloud registration in Section 2, we explain our methodology in Section 3. Subsequently, in Section 4, we provide a theoretical consideration of the new measure for point quality assessment and briefly discuss the consequences for reliable range measurements in this context. This is followed by a presentation of experimental results in Section 5 and a discussion of these results in Section 6. Finally, in Section 7, we provide concluding remarks and suggestions for future work.

\section{RELATED WORK}

In the following, we reflect the related work and thereby focus on how to quantify the quality of each range measurement (Section 2.1), how to extract suitable features (Section 2.2) and how to efficiently register TLS point clouds (Section 2.3).

\subsection{Point Quality Assessment}

Generally, the accuracy of a range measurement depends on the design of the measurement system in terms of angular accuracy, range accuracy and resolution and, additionally, on the characteristics of the observed scene in terms of scanning geometry (i.e. the distance and orientation of scanned surfaces), object edges, surface reflectivity and environmental conditions (Hebert and Krotkov, 1992; Boehler et al., 2003; Soudarissanane et al., 2011). While a systematic error modeling may account for a variety of potential error sources contributing to the uncertainty of range measurements (Lichti et al., 2005; Lichti and Licht, 2006; Barber et al., 2008; Boström et al., 2008), scene-specific issues cannot be generalized and therefore have to be treated differently. 
For this reason, we purely focus on filtering raw point cloud data by exploiting the captured information. On the one hand, a simple approach for filtering may be based on the measured intensity information (Barnea and Filin, 2007), since very low intensity values are likely to correspond to unreliable range measurements. On the other hand, it seems advisable to filter points at depth discontinuities as these exhibit the largest distance error. A respective filtering may for instance be achieved by involving the scan line approximation technique (Fuchs and May, 2008), by applying the Laplacian operator on the range image (Barnea and Filin, 2008) or by considering the standard deviation of range values within a local patch of the range image (Weinmann and Jutzi, 2011). Accounting for both intensity and range information, the combination of removing points with low values in the intensity image as well as points at edges in the range image has been proposed in order to obtain an adequate 3D representation of a scene (Swadzba et al., 2007). Furthermore, it seems to be advisable to take into account that the scanning geometry w.r.t. the incidence angle (i.e. the angle between incoming laser beam and surface normal) may have a significant influence on the accuracy of a range measurement which becomes visible by an increase in measurement noise with increasing incidence angles (Soudarissanane et al., 2011). Accordingly, it seems to be desirable to have a common and generic measure which considers reliability in terms of both object edges and incidence angle.

\subsection{Feature Extraction}

Nowadays, the most accurate alignment of TLS data is still obtained via manipulating the observed scene by placing artificial markers which represent clearly demarcated corresponding points in different scans. Thus, such markers may easily be extracted either manually or automatically (Akca, 2003; Franaszek et al., 2009). Even though a good quality of the registration process is ensured, this procedure may however be rather time-consuming particularly for a large number of scans - and it hence often tends to be intractable. Consequently, a fully automated registration of scans without using artificial markers is desirable.

Generally, an automated procedure for point cloud registration may be based on the full point clouds and applying standard techniques such as the Iterative Closest Point (ICP) algorithm (Besl and McKay, 1992) or Least Squares 3D Surface Matching (Gruen and Akca, 2005) which exploit the spatial 3D information in order to minimize either the difference between point clouds or the distance between matched surfaces. Since these standard techniques typically result in a higher computational burden, it seems advisable to extract relevant information in the form of specific features from the point clouds in order to alleviate point cloud registration. Such relevant information may for instance be derived from the distribution of the points within each point cloud by using the normal distributions transform (NDT) either on 2D scan slices (Brenner et al., 2008) or in 3D (Magnusson et al., 2007). Furthermore, the detection of corresponding features may be based on specific 3D structures in the scene which can be characterized by geometric primitives such as planar structures (Brenner et al., 2008; Pathak et al., 2010; Theiler et al., 2012) and/or even more complex primitives such as spheres, cylinders and tori (Rabbani et al., 2007). Additionally, lines resulting from the intersection of neighboring planar structures or the boundary of a planar structure could be involved for the registration process (Stamos and Leordeanu, 2003). However, all these feature types representing specific geometric primitives encounter significant challenges in case of scene symmetry and they are not suited in scenes without regular surfaces.

Facing general scenes where we may not assume the presence of specific 3D shape primitives, the least assumptions are possible when focusing on point-like features. Such features may for instance be based on geometric curvature or normal vectors of the local surface (Bae and Lichti, 2008), or on the application of interest point detectors in 3D space, e.g. via a 3D Harris corner detector or a 3D Difference-of-Gaussians (DoG) detector (Theiler et al., 2013; Theiler et al., 2014). However, in order to increase computational efficiency and furthermore account for the fact that - due to the use of a line-of-sight instrument with a specific angular resolution - a significant variation of the point density may be expected, most of the proposed 3D interest point detectors are based on a voxelization of the scene and thus strongly depend on the selected voxel size.

Taking into account that the range information is acquired for points on a regular scan grid, we may easily derive an image representation in the form of range images and then extract interest points from these range images, e.g. by applying a min-max algorithm (Barnea and Filin, 2008), the Harris corner detector (Steder et al., 2009), the Laplacian-of-Gaussian (LoG) detector (Steder et al., 2010) or the Normal Aligned Radial Feature (NARF) detector (Steder et al., 2011). While the design of an interest point detector may principally also account for finding keypoints on geometrically smooth 3D surfaces, such approaches generally require characteristic 3D structures in the scene which may not always be that well-distributed in larger distances to the sensor.

Since modern scanning devices also allow to acquire intensity information on the discrete scan grid and thus intensity images which typically provide complementary information with a higher level of distinctiveness than range images (Seo et al., 2005), some approaches for point cloud registration involve features in the form of keypoints extracted from intensity images derived from reflectance data (Boehm and Becker, 2007; Wang and Brenner, 2008; Kang et al., 2009; Alba et al., 2011; Weinmann et al., 2011) or co-registered camera images (Al-Manasir and Fraser, 2006; Barnea and Filin, 2007; Wendt, 2007). The respective forwardprojection of such 2D keypoints w.r.t. the corresponding range information allows to derive sparse point clouds of (almost) identical 3D points and thus significantly alleviates point cloud registration while improving computational efficiency.

\subsection{Point Cloud Registration}

Nowadays, most of the approaches for aligning pairs of overlapping scans exploit a keypoint-based point cloud registration which has proven to be among the most efficient strategies. While it has recently been proposed to exploit the spatial arrangement of 3D keypoints for a geometrical constraint matching (Theiler et al., 2013; Theiler et al., 2014), still most investigations involve forward-projected 2D keypoints detected in image representations of the captured intensity or range information. This may be motivated by the fact that feature correspondences may easily and efficiently be derived by comparing keypoint descriptors. The forward-projection of corresponding 2D keypoints to 3D space, in turn, results in sparse point clouds which may for instance be aligned by estimating a standard rigid transformation (Arun et al., 1987; Horn et al., 1988; Eggert et al., 1997). Since some feature correspondences might represent outliers, a robust estimation by involving the RANSAC algorithm (Fischler and Bolles, 1981) is typically exploited (Seo et al., 2005; Boehm and Becker, 2007; Barnea and Filin, 2007).

A different strategy for point cloud registration consists of projecting scans with the associated intensity information onto the image planes of virtual cameras and minimizing discrepancies in color, range and silhouette between pairs of images (Pulli et al., 2005). While this is rather impractical for large point clouds 
and thus for the registration of TLS scans, a more efficient approach has recently been proposed with keypoint-based projective scan matching (Weinmann et al., 2011; Weinmann and Jutzi, 2011), where forward-projected 2D keypoints are back-projected onto the image plane of a virtual camera in order to derive $3 \mathrm{D} / 2 \mathrm{D}$ correspondences which, in turn, serve as input for a registration scheme involving the Efficient-Perspective- $n$-Point $(\mathrm{EP} n \mathrm{P})$ algorithm (Moreno-Noguer et al., 2007) and the RANSAC algorithm (Fischler and Bolles, 1981), and this scheme delivers highly accurate registration results for coarse registration. Such a strategy not only involves 3D cues based on the point clouds, but also 2D cues based on imagery and hence the results for coarse and fine registration may partially be in the same range.

Finally, it may be desirable to derive a measure describing the similarity of different scans which may efficiently be exploited in order to automatically organize a given set of unorganized scans for a successive pairwise registration. This is particularly important for approaches relying on the use of range and intensity images, since a higher overlap of considered scans results in a higher similarity and thus more feature correspondences which, in turn, increases the robustness of respective registration approaches. In this regard, it has been proposed to derive a topological graph, where the nodes represent the single scans and the edges describe their similarity, e.g. based on the number of matched lines determined from the range information (Stamos and Leordeanu, 2003) or the number of point correspondences between respective intensity images (Weinmann and Jutzi, 2011). The smaller the weight of an edge, the smaller the overlap between the scans corresponding to the nodes connected by this edge. Thus, an appropriate scan order for a reliable successive pairwise registration may be derived via a minimum spanning tree (Huber and Hebert, 2003).

\section{METHODOLOGY}

Our proposed methodology for a pairwise registration of TLS scans consists of three components which are represented by point quality assessment (Section 3.1), feature extraction (Section 3.2) and point cloud registration (Section 3.3)

\subsection{Point Quality Assessment}

Generally, a filtering of raw point cloud data in terms of removing $3 \mathrm{D}$ points corresponding to unreliable range measurements may be based on intensity information (Barnea and Filin, 2007), since low intensity values typically indicate unreliable range measurements. However, such considerations do not account for edge effects where noisy range measurements are likely to occur although the respective intensity values might be reasonable. Hence, we focus on two strategies which are based on the geometric measures of $(i)$ range reliability and (ii) planarity for quantifying the quality of a range measurement.

3.1.1 Range Reliability: The first measure of range reliability (Weinmann and Jutzi, 2011) is motivated by the fact that a laser beam has certain physical dimensions. Thus, the projection of a laser beam on the target area results in a laser footprint, i.e. a spot with finite dimension, that may vary depending on the slope of the local surface and material characteristics (Vosselman and Maas, 2010). Consequently, if a measured 3D point corresponds to a footprint on a geometrically smooth surface, the captured range information is rather reliable when assuming Lambertian surfaces and reasonable incidence angles. However, at edges of objects, a footprint may cover surfaces at different distances to the sensor, and thus the captured range information is rather unreliable. Even more critical are range measurements corresponding to the sky, since these mainly arise from atmospheric effects.
In order to remove unreliable range measurements - which typically appear as noisy behavior in a point cloud - it has been proposed to quantify range reliability by considering a local image patch for each point on the regular $2 \mathrm{D}$ grid and assigning the standard deviation $\sigma_{r, 3 \times 3}$ of all range values within a $(3 \times 3) \mathrm{im}$ age neighborhood to the respective center point. Deriving $\sigma_{r, 3 \times 3}$ for all pixels of the 2D representation yields a confidence map, and a simple thresholding is sufficient to distinguish reliable measurements from unreliable ones. More specifically, low values $\sigma_{r, 3 \times 3}$ indicate a 3D point on a smooth surface and are therefore assumed to be reliable, whereas high values $\sigma_{r, 3 \times 3}$ indicate noisy and unreliable range measurements. For the separation between reliable and unreliable range measurements, a predefined threshold $t_{\sigma}=0.03 \ldots 0.10 \mathrm{~m}$ has been proposed (Weinmann and Jutzi, 2011). An example demonstrating the effect of such a point cloud filtering is given in Figure 1 for a part of a terrestrial laser scan which corresponds to $2304 \times 1135$ scanned 3D points and has been acquired with a Leica HDS6000 on the KIT campus in Karlsruhe, Germany. The suitability of such an approach has been demonstrated for data captured with a laser scanner (Weinmann and Jutzi, 2011) and for data captured with a range camera (Weinmann et al., 2013), but the manual selection of a threshold based on prior knowledge on the scene represents a limitation.

3.1.2 Planarity: The second measure - which we propose in this paper - is motivated by the fact that reliable range information typically corresponds to almost planar structures in the scene. Consequently, we aim to quantify planarity for each pixel of the $2 \mathrm{D}$ representation by considering local image patches. In analogy to the measure of range reliability, we consider $(3 \times 3)$ image neighborhoods as local image patches in order to assign a measure of planarity to the respective center point. From the spatial $X Y Z$-coordinates of all 3D points corresponding to the pixels in the $(3 \times 3)$ image neighborhood, we derive the $3 \mathrm{D}$ covariance matrix known as $3 \mathrm{D}$ structure tensor $\mathbf{S} \in \mathbb{R}^{3 \times 3}$ whose eigenvalues $\lambda_{1}, \lambda_{2}, \lambda_{3} \in \mathbb{R}$ with $\lambda_{1} \geq \lambda_{2} \geq \lambda_{3} \geq 0$ are further exploited in order to define the dimensionality features of linearity $L_{\lambda}$, planarity $P_{\lambda}$ and scattering $S_{\lambda}$ (West et al., 2004):

$$
L_{\lambda}=\frac{\lambda_{1}-\lambda_{2}}{\lambda_{1}} \quad P_{\lambda}=\frac{\lambda_{2}-\lambda_{3}}{\lambda_{1}} \quad S_{\lambda}=\frac{\lambda_{3}}{\lambda_{1}}
$$

These dimensionality features are normalized by $\lambda_{1}$, so that they sum up to 1 and the largest value among the dimensionality features indicates the characteristic behavior assigned to the respective pixel. Accordingly, a pixel represents a planar 3D structure and thus rather reliable range information if the constraint

$$
P_{\lambda, 3 \times 3}:=P_{\lambda} \geq \max \left\{L_{\lambda}, S_{\lambda}\right\}
$$

is satisfied. Note that - in contrast to the measure of range reliability - this definition for reliability is fully generic without involving any manually specified thresholds and thus prior knowledge on the scene. Some results when applying the proposed measure for point cloud filtering are illustrated in Figure 2.

3.1.3 A Comparison of Different Measures: In order to provide an impression on the performance of the different measures for quantifying the quality of range measurements, the derived binary confidence maps for $(i)$ intensity values above a threshold of $t_{I}=10$ (w.r.t. a gray-valued image of type uint8), (ii) the measure of range reliability when applying a threshold of $t_{\sigma}=0.03 \mathrm{~m}$, and (iii) the proposed generic measure of planarity are depicted in Figure 3 and the corresponding effect in 3D space is visualized in Figure 4. These figures clearly reveal that the use of intensity information alone is not sufficient to adequately filter point cloud data and thereby completely remove the noisy behavior. In contrast, the strategies based on the two geometric 

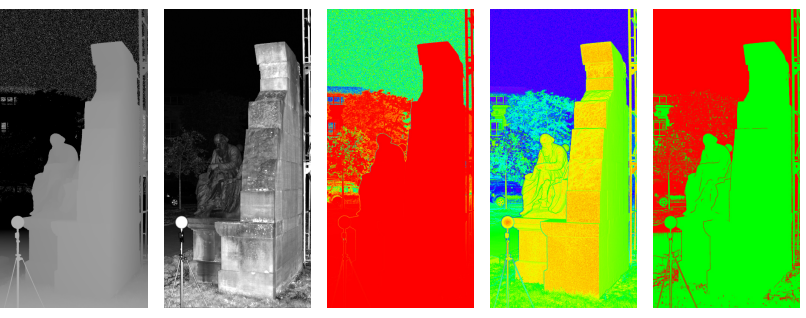

Figure 1. Range image, intensity image, visualization of range reliability, logarithmic representation of range reliability and the binary confidence map derived by thresholding based on a manually selected threshold of $t_{\sigma}=0.03 \mathrm{~m}$ (from left to right).

measures retain adequate representations of local object surfaces. Whereas the strategy based on the measure of range reliability provides almost planar object surfaces for significantly varying incidence angles, the strategy based on the measure of planarity only provides almost perpendicular object surfaces with almost planar behavior and thus favors lower incidence angles which tend to yield more accurate range measurements (Figure 3)

\subsection{Feature Extraction}

Once we are able to quantify the quality of a range measurement, the next step consists of deriving correspondences between the respective scans. For this purpose, we consider the derived $2 \mathrm{D}$ image representations. As intensity images typically provide a higher level of distinctiveness than range images (Seo et al., 2005) and thus also contain information about the local environment which is not represented in range images, it is advisable to involve these intensity images for finding corresponding information between different scans. Among a variety of visual features (Weinmann, 2013), local features seem to be favorable as they may be localized accurately with efficient feature detectors and as they remain stable for reasonable changes in viewpoint (Tuytelaars and Mikolajczyk, 2008). Characterizing such a local feature by deriving a feature descriptor from the local image neighborhood even allows an individual identification of local features across different images. Thus, using local features has become very popular for a wide range of applications.

As one of the most powerful approaches for extracting local features, we apply the Scale Invariant Feature Transform (SIFT) (Lowe, 2004) on the intensity images. This yields distinctive keypoints at $2 \mathrm{D}$ image locations $\mathbf{x} \in \mathbb{R}^{2}$ as well as the respective local descriptors which are invariant to image scaling and image rotation, and robust w.r.t. image noise, changes in illumination and reasonable changes in viewpoint. In order to reject ambiguous matches, the descriptors extracted for keypoints in different images $\mathcal{I}_{i}$ and $\mathcal{I}_{j}$ are not simply compared via their Euclidean distance, but via the ratio of the Euclidean distances of a descriptor belonging to a keypoint in $\mathcal{I}_{i}$ to the nearest neighbor and the second nearest neighbor in $\mathcal{I}_{j}$. A low value of this ratio indicates a high similarity to only one of the derived descriptors belonging to $\mathcal{I}_{j}$, whereas a high value indicates that the nearest and second nearest neighbor are quite similar. Consequently, the ratio describes the distinctiveness of the matched features, and it is selected to be below a certain threshold $t_{\mathrm{SIFT}}$ which is typically chosen within the interval $[0.6,0.8]$ for obtaining reliable feature correspondences $\mathbf{x}_{i} \leftrightarrow \mathbf{x}_{j}$ between images $\mathcal{I}_{i}$ and $\mathcal{I}_{j}$.

\subsection{Point Cloud Registration}

Generally, the forward-projection of the extracted 2D keypoints w.r.t. the corresponding range information yields sparse point
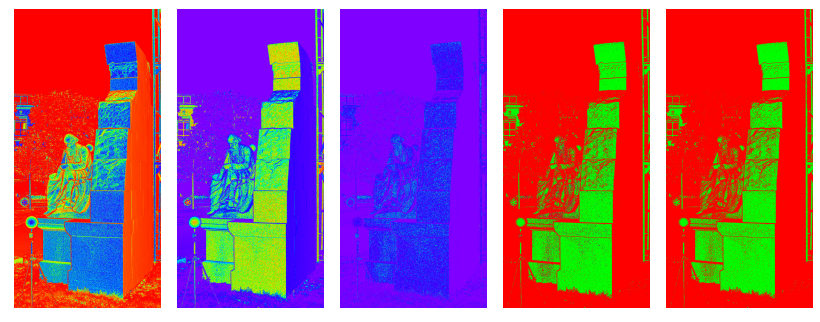

Figure 2. Visualization for linearity $L_{\lambda}$, planarity $P_{\lambda}$, scattering $S_{\lambda}$, the classification of each pixel according to its local behavior (linear: red; planar: green; scattered: blue) and the derived binary confidence map (from left to right).
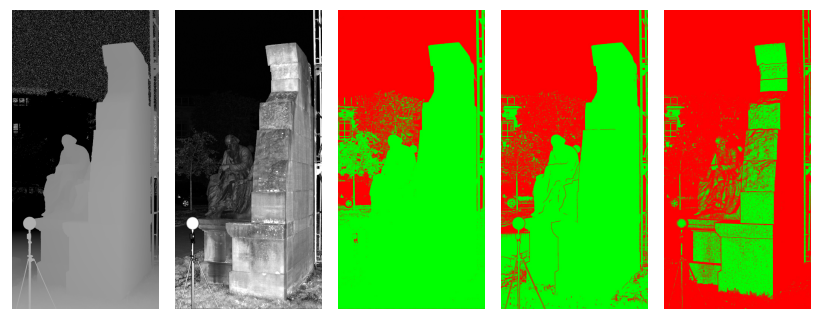

Figure 3. Range image, intensity image and the derived binary confidence maps based on intensity, range reliability and planarity (from left to right).

clouds, where typically a high percentage of the detected feature correspondences indicates physically (almost) identical 3D points. As SIFT features are localized with subpixel accuracy, the respective spatial information has to be interpolated from the information available for the regular and discrete 2D grid, e.g. by applying a bilinear interpolation. Instead of involving only $3 \mathrm{D}$ cues as for instance done when estimating a standard rigid transformation, we involve both 3D and 2D cues for keypointbased point cloud registration in analogy to recent investigations on projective scan matching (Weinmann et al., 2011; Weinmann and Jutzi, 2011) as such a strategy provides both computational efficiency and robustness to outlier correspondences.

Without loss of generality, we may assume that - when considering a scan pair $\mathcal{P}_{i}=\left\{\mathcal{S}_{i}, \mathcal{S}_{j}\right\}$ - the position and orientation of scan $\mathcal{S}_{i}$ is known w.r.t. the world coordinate frame. Consequently, for scan $\mathcal{S}_{i}$, the respective forward-projection of 2D keypoints $\mathbf{x}_{i} \in \mathbb{R}^{2}$ results in $3 \mathrm{D}$ coordinates $\mathbf{X}_{i} \in \mathbb{R}^{3}$ which are also known w.r.t. the world coordinate frame, whereas the forward-projection of $2 \mathrm{D}$ keypoints $\mathbf{x}_{j} \in \mathbb{R}^{2}$ results in $3 \mathrm{D}$ coordinates $\mathbf{X}_{j} \in \mathbb{R}^{3}$ which are only known w.r.t. the local coordinate frame of the sensor for scan $\mathcal{S}_{j}$. The basic idea of projective scan matching consists of introducing $2 \mathrm{D}$ cues by back-projecting the $3 \mathrm{D}$ points $\mathbf{X}_{j}$ onto a virtual image plane for which the projection model of a pinhole camera is exploited (Weinmann et al., 2011):

$$
\mathbf{x}_{j}^{*}=\mathbf{K}[\mathbf{R} \mid \mathbf{t}] \mathbf{X}_{j}
$$

In this equation, the calibration matrix of the virtual camera is denoted with $\mathbf{K}$ and arbitrary parameters may be selected for specifying focal lengths and principal point (Weinmann et al., 2011; Weinmann and Jutzi, 2011). Furthermore, the rotation matrix $\mathbf{R}$ and the translation vector $\mathbf{t}$ describe the relative orientation of the virtual camera w.r.t. the local coordinate frame of the laser scanner, and they are specified in a way that the virtual camera looks into the horizontal direction and that the position of the virtual camera coincides with the location of the laser scanner, i.e. $\mathbf{t}=\mathbf{0}$. The points $\mathbf{x}_{j}^{*} \in \mathbb{R}^{2}$, in turn, allow to transform $n$ feature correspondences $\mathbf{x}_{i, k} \leftrightarrow \mathbf{x}_{j, k}$ with $k=1, \ldots, n$ to $3 \mathrm{D} / 2 \mathrm{D}$ corre- 

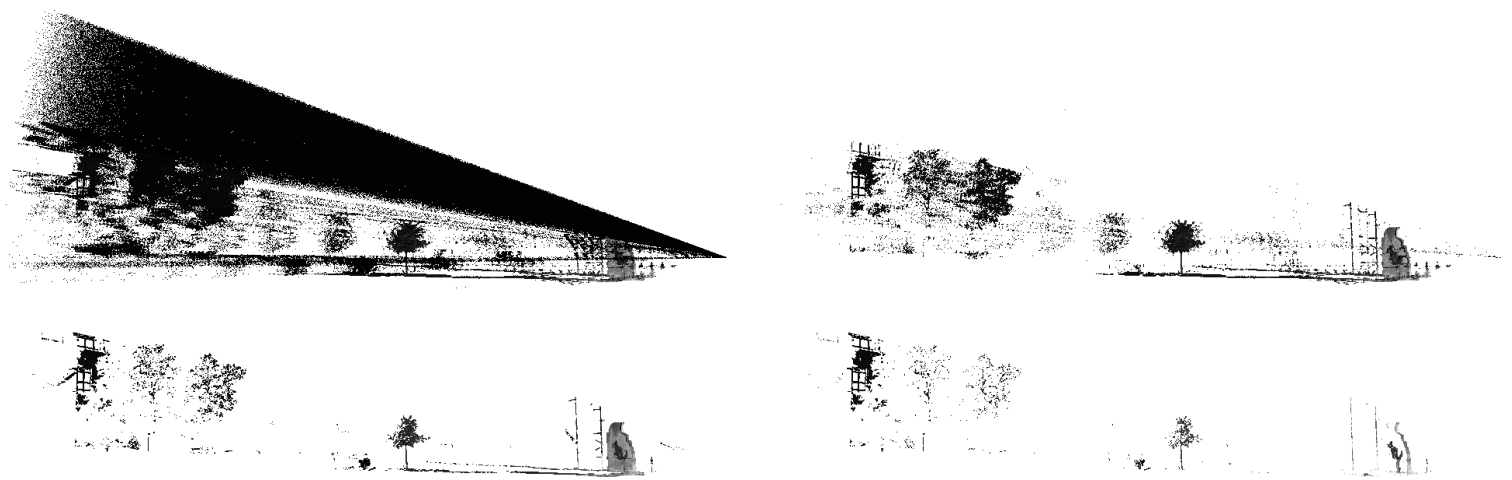

Figure 4. Raw point cloud data (top left), point cloud data filtered via intensity information (top right), point cloud data filtered via the measure of range reliability (bottom left) and point cloud data filtered via the measure of planarity (bottom right).

spondences $\mathbf{X}_{i, k} \leftrightarrow \mathbf{x}_{j, k}^{*}$ and thus to relate the task of point cloud registration to the task of solving the well-known Perspective- $n$ Point $(\mathrm{P} n \mathrm{P})$ problem, where the aim is to estimate the exterior orientation or pose of a camera from a set of $n$ correspondences between 3D points $\mathbf{X}_{i, k}$ of a scene and their 2D projections $\mathbf{x}_{j, k}^{*}$ in the image plane of a camera (Fischler and Bolles, 1981).

A robust approach for solving the $\mathrm{P} n \mathrm{P}$ problem has been proposed with the Efficient Perspective- $n$-Point $(\mathrm{EP} n \mathrm{P})$ algorithm (Moreno-Noguer et al., 2007) which represents a non-iterative method and provides an accurate solution to the $\mathrm{P} n \mathrm{P}$ problem with only linear complexity. Compared to other approaches for solving the $\mathrm{P} n \mathrm{P}$ problem, this algorithm is not only fast and accurate, but also designed to work with a large number of correspondences and it does not require an initial estimate. The EP $n \mathrm{P}$ algorithm is based on the idea of expressing the $n$ known 3D scene points $\mathbf{X}_{i}$ as a weighted sum of four virtual and non-coplanar control points $\mathbf{C}_{j} \in \mathbb{R}^{3}$ for general configurations. Denoting the involved weights as $\alpha_{i j}$ and introducing a superscript ${ }^{c}$ which indicates coordinates in the camera coordinate frame, each $3 \mathrm{D} / 2 \mathrm{D}$ correspondence provides a relation of the form

$$
w_{i}\left[\begin{array}{c}
\mathbf{x}_{i} \\
1
\end{array}\right]=\mathbf{K} \mathbf{X}_{i}^{c}=\mathbf{K} \sum_{j=1}^{4} \alpha_{i j} \mathbf{C}_{j}^{c}
$$

where $\mathbf{K}$ describes the calibration matrix, in our case the one of the virtual camera. Considering the respective three equations, the scalar projective parameters $w_{i}$ can be determined according to the third equation and substituted into the other two equations. Concatenating the two modified equations for $i=1, \ldots, n$ yields a linear equation system $\mathbf{M} \mathbf{y}=\mathbf{0}$, where $\mathbf{y}$ contains the $3 \mathrm{D}$ coordinates of the four control points $\mathbf{C}_{j}$. For more details on the efficient solution of this equation system, we refer to the original paper (Moreno-Noguer et al., 2007). Once both world and camera coordinates of the $3 \mathrm{D}$ points are known, the transformation parameters aligning both coordinate frames can be retrieved via standard methods involving a closed-form solution in the leastsquares sense (Horn et al., 1988; Arun et al., 1987).

For a robust estimation in case of existing outlier correspondences, the RANSAC algorithm (Fischler and Bolles, 1981) represents the method of choice as it eliminates the influence of outlier correspondences which are not in accordance with the largest consensus set supporting the given transformation model. Following the original implementation (Moreno-Noguer et al., 2007), the RANSAC-based $\mathrm{EP} n \mathrm{P}$ scheme relies on selecting small, but not minimal subsets of seven correspondences for estimating the model parameters and checking the whole set of correspondences for consistent samples. In comparison to minimal subsets, this further reduces the sensitivity to noise. In order to avoid testing all possible subsets, which would be very time-consuming, we exploit an efficient variant, where the number of iterations - which equals the number of randomly chosen subsets - is selected high enough, so that a subset including only inlier correspondences is selected with a certain probability $p$ (Fischler and Bolles, 1981; Hartley and Zisserman, 2008).

Finally, we conduct a geometric outlier removal based on 3D distances and an ICP-based fine registration (Weinmann et al., 2011; Wang and Brenner, 2008).

\section{PLANARITY VS. RANGE RELIABILITY}

In this section, we carry out theoretical considerations for the proposed measure of planarity and thereby point out consequences concerning what we may expect when applying this measure on range images. This is of utmost importance since we may thus easily explain the significant differences between the binary confidence maps depicted in Figure 1 and Figure 2.

In order to verify the suitability of the proposed measure of planarity, we consider fundamentals of projective geometry as for instance described in (Hartley and Zisserman, 2008). Generally, the $3 \mathrm{D}$ coordinates of a point $\mathbf{X} \in \mathbb{R}^{3}$ on a ray in $3 \mathrm{D}$ space satisfy the constraint $\mathbf{X}=\mathbf{A}+b \mathbf{v}$, where $\mathbf{A} \in \mathbb{R}^{3}$ denotes a known point on the ray, $b \in \mathbb{R}$ represents a scalar factor and $\mathbf{v} \in \mathbb{R}^{3}$ indicates the direction of the ray. Without loss of generality, we may transfer this equation to camera coordinates as indicated by a superscript ${ }^{c}$, i.e. $\mathbf{X}^{c}=\mathbf{A}^{c}+b \mathbf{v}^{c}$. Since - when assuming the model of a pinhole camera - the considered rays intersect each other at the projective center $\mathbf{0}^{c}$, we may use the point $\mathbf{A}^{c}=\mathbf{0}^{c}=[0,0,0]^{T}$ as known point on all rays. Furthermore, we may exploit the definition of the camera coordinate frame (where $X^{c}$ points to the right, $Y^{c}$ to the bottom and $Z^{c}$ in depth). Looking along the $Z^{c}$-axis and assuming an angular resolution $\alpha$ of the camera, the directions $\mathbf{v}^{c}$ of the 8 neighboring rays which are exploited to obtain a local $(3 \times 3)$ image neighborhood can easily be derived by intersection with the $\left(Z^{c}=1\right)$-plane. Thus, we evaluate the geometric behavior of range measurements in a field-of-view given by $(2 \alpha \times 2 \alpha)$.

For our example, we assume that the 9 defined rays characterizing a local $(3 \times 3)$ image neighborhood intersect a plane $\boldsymbol{\pi}$ which is parameterized in the camera coordinate frame by a point $\mathbf{X}_{\pi}^{c}$ and a normal vector $\mathbf{n}_{\pi}^{c}$. Thereby, we define the point $\mathbf{X}_{\pi}^{c}$ as the point which results from the intersection of $\pi$ with the $Z^{c}$-axis, and we further assume that the distance between $\mathbf{X}_{\boldsymbol{\pi}}^{c}$ and $\mathbf{0}^{c}$ is given by $d$, i.e. $\mathbf{X}_{\pi}^{c}=[0,0, d]^{T}$. Initially, we consider the case of a normal vector $\mathbf{n}_{\pi}^{c}$ which coincides with the $Z^{c}$-axis, and thus the plane $\pi$ is parallel to the $X^{c} Y^{c}$-plane. Subsequently, we rotate the plane $\boldsymbol{\pi}$ by an angle $\beta$ around the axis defined by the point $\mathbf{X}_{\boldsymbol{\pi}}^{c}=[0,0, d]^{T}$ and the direction $[0,1,0]^{T}$. 


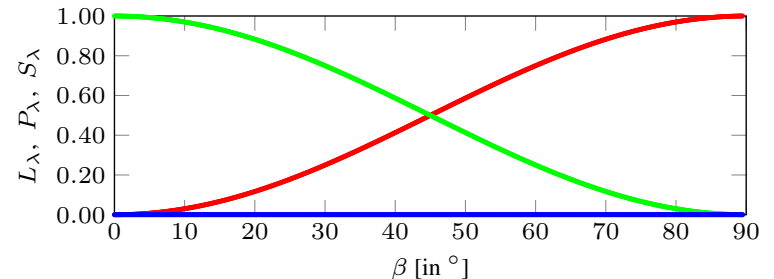

Figure 5. Behavior of the dimensionality features of linearity $L_{\lambda}$ (red), planarity $P_{\lambda}$ (green) and scattering $S_{\lambda}$ (blue) for increasing incidence angles $\beta$. Note that the synthetic data is not corrupted with noise.

From the 9 points of intersection, we exploit the 3D coordinates in order to derive the 3D structure tensor $\mathbf{S}$ and its eigenvalues $\lambda_{1}$, $\lambda_{2}$ and $\lambda_{3}$ as well as the dimensionality features of linearity $L_{\lambda}$, planarity $P_{\lambda}$ and scattering $S_{\lambda}$ (cf. Section 3.1.2). For an example which is close to the scenario when using a $3 \mathrm{D}$ range camera, we select the angular resolution to $\alpha=0.2^{\circ}$ and the distance between projective center and $\mathbf{X}_{\boldsymbol{\pi}}^{c}$ to $d=5 \mathrm{~m}$. The respective values of the dimensionality features for angles $\beta \in\left[0^{\circ}, 90^{\circ}\right]$ are depicted in Figure 5, and they reveal that the locally planar 3D structure provides a planar behavior in the interval $\left[0^{\circ}, 45^{\circ}\right]$ and a linear behavior beyond this interval. As a consequence, range measurements are assumed to be reliable if the local $(3 \times 3) \mathrm{im}$ age neighborhood represents a locally planar 3D structure with an incidence angle in $\left[0^{\circ}, 45^{\circ}\right]$. Note that, due to the narrow fieldof-view of $(2 \alpha \times 2 \alpha)$ for a local $(3 \times 3)$ image patch, noisy range measurements $e . g$. corresponding to the sky will not be indicated by a scattered behavior, but by a linear behavior since only a significant variation in ray direction will be present.

For a comparison to the measure $\sigma_{r, 3 \times 3}$ of range reliability ( $c f$. Section 3.1.1), we provide the respective behavior of $\sigma_{r, 3 \times 3}$ for the same example in Figure 6. The considered range values are represented by the distance between the projection center $\mathbf{0}^{c}$ and those points resulting from the intersection of the defined rays with the plane $\boldsymbol{\pi}$, and $\sigma_{r, 3 \times 3}$ is derived as the respective standard deviation of these range values. Applying the proposed threshold of $t_{\sigma}=0.03 \mathrm{~m}$, range measurements are assumed to be reliable for incidence angles of less than about $63.3^{\circ}$. A threshold of $t_{\sigma}=0.10 \mathrm{~m}$ even results in reliable range measurements up to incidence angles of about $81.4^{\circ}$. Consequently, the binary confidence map shown in Figure 1 indicates more planar surfaces which are assumed to provide reliable range measurements than the binary confidence map depicted in Figure 2, where only planar surfaces with incidence angles up to about $45^{\circ}$ are assumed to provide reliable range measurements.

\section{EXPERIMENTAL RESULTS}

For demonstrating the performance of our methodology, we involve a standard TLS benchmark dataset (Section 5.1) and describe the conducted experiments as well as the respective results (Section 5.2).

\subsection{Dataset}

In order to allow a comparison of other approaches to our results, we demonstrate the performance of our methodology on the Holzmarkt dataset which represents a publicly available TLS benchmark dataset ${ }^{1}$. This dataset has been acquired in an urban environment with a Riegl LMS-Z360i laser scanner, and it consists of 12 upright and 8 tilted scans with given reference values for the relative orientation. For our experiments, we use the

${ }^{1}$ The Holzmarkt dataset is available at http://www.ikg.unihannover.de/index.php?id=413\&L=de (accessed: 30 March 2015)

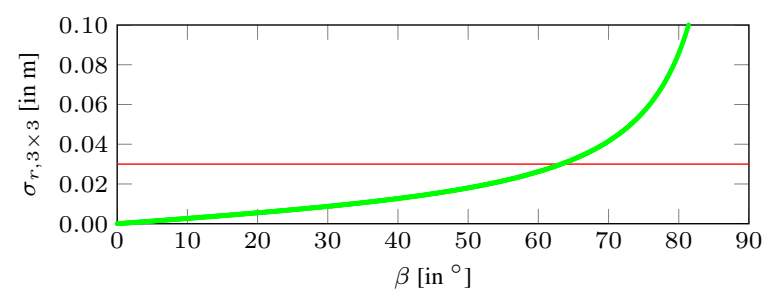

Figure 6. Behavior of the measure $\sigma_{r, 3 \times 3}$ of range reliability for increasing incidence angles $\beta$. The applied threshold of $0.03 \mathrm{~m}$ is indicated with a red line. Note that the synthetic data is not corrupted with noise.

upright scans, where each scan covers $360^{\circ}$ in the horizontal direction and $90^{\circ}$ in the vertical direction with a single shot measurement accuracy of $12 \mathrm{~mm}$ and an angular resolution of $0.12^{\circ}$ up to a range of approximately $200 \mathrm{~m}$ (Wang and Brenner, 2008). Thus, each scan is represented by 2.25 million 3D points at a regular scan grid of $3000 \times 750$ points. Since both range and intensity information are available for each point on the scan grid, $2 \mathrm{D}$ representations in the form of panoramic range and intensity images may easily be derived.

\subsection{Experiments}

First, we sort the scans w.r.t. their similarity in order to provide the basis for a successive pairwise registration. For this purpose, we exploit a minimum spanning tree based on the number of feature correspondences between the different intensity images (Weinmann and Jutzi, 2011). As a result, we obtain ordered scans $\mathcal{S}_{i}$. The whole procedure takes approximately $607.04 \mathrm{~s}$ for the given set of 12 scans on a standard desktop computer (Intel Core2 Quad Q9550, 2.83GHz, 8GB RAM, Matlab implementation).

Subsequently, we successively conduct pairwise registration via the RANSAC-based EP $n \mathrm{P}$ scheme and thereby involve the different methods for point quality assessment for removing unreliable feature correspondences $\left(t_{I}=10, t_{\sigma}=0.1 \mathrm{~m}, t_{\mathrm{SIFT}}=0.66\right)$. Since the random sampling may lead to slightly different estimates, we average all position and angle estimates over 20 runs. For the different scan pairs $\mathcal{P}_{i}=\left\{\mathcal{S}_{i}, \mathcal{S}_{i+1}\right\}$ with $i=1, \ldots, 11$, the remaining errors after coarse and fine registration are shown in Figure 7 as well as the achieved improvement. These results reveal that already the step of coarse registration provides accurate position estimates, where the position error indicating the absolute deviation of the estimated scan position from the reference data is less than $5 \mathrm{~cm}$ for almost all cases. After fine registration, the remaining position error is in the range between $0.47 \mathrm{~cm}$ and $4.10 \mathrm{~cm}$. The respective angle errors are in the interval between $0.0001^{\circ}$ and $0.2845^{\circ}$ after coarse registration, and they are reduced to the interval between $0.0002^{\circ}$ and $0.0919^{\circ}$ after fine registration.

In order to obtain an impression on the computational effort for pairwise registration, the mean processing times required for the different subtasks are listed in Table 1 . Since those processing times for coarse registration vary significantly when involving different methods for point quality assessment, a respective visualization is provided in Figure 8. Based on these numbers, in total, a processing time of $191.35 \mathrm{~s}$ may be expected in the worst case for a pairwise registration of the considered scans.

\section{DISCUSSION}

When having a closer look on the results after coarse registration (Figure 7, top), we can observe a relatively good position estimate except for the last scan pair $\mathcal{P}_{11}$. This however results from 

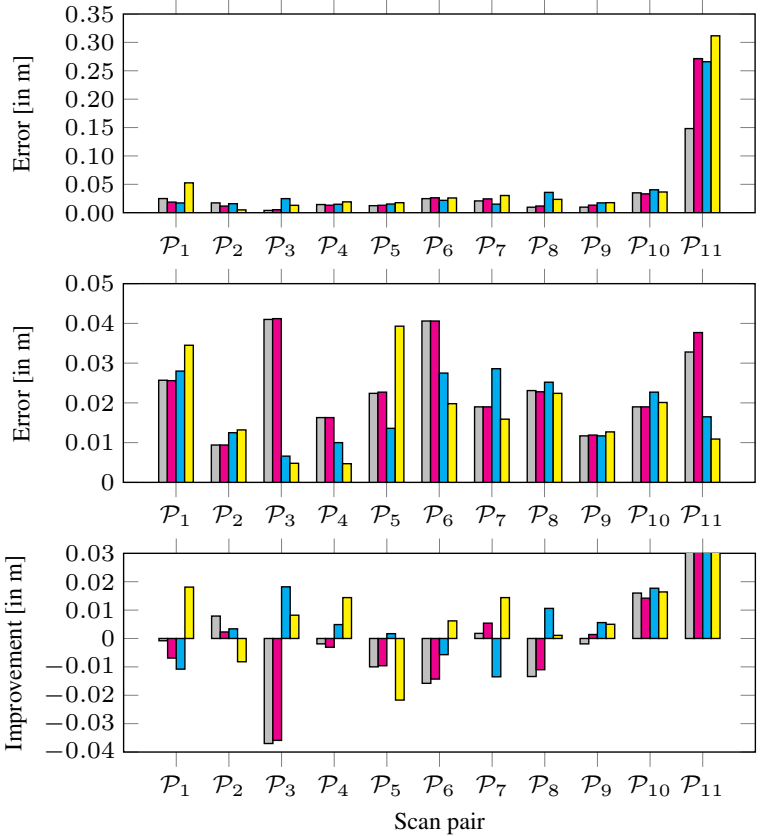

Figure 7. Mean position error after coarse registration (top), after fine registration (center) and the respective improvement (bottom) for the scan pairs $\mathcal{P}_{i}=\left\{\mathcal{S}_{i}, \mathcal{S}_{i+1}\right\}$ when applying no reliability check (gray) and when applying reliability checks w.r.t. intensity (magenta), range reliability (cyan) or planarity (yellow).

\begin{tabular}{lrr}
\hline Task & Number of executions & Processing time [in s] \\
\hline Point quality assessment & 2 & $<0.01$ \\
$\quad$-Intensity & & 1.32 \\
$\quad$-Range Reliability & & 78.39 \\
$\quad$-Planarity & 2 & 8.77 \\
Feature extraction & 2 & 0.17 \\
Forward projection & 1 & 0.21 \\
Feature matching & 1 & $0.10 \ldots .37$ \\
Coarse registration & 1 & $<0.04$ \\
Outlier removal & 1 & $<0.07$ \\
Fine registration & & \\
\hline
\end{tabular}

Table 1. Required processing times for different subtasks of a pairwise registration.

the fact that the distance between the respective scans is about $4 \ldots 6 \mathrm{~m}$ for scan pairs $\mathcal{P}_{1}, \ldots, \mathcal{P}_{10}$, whereas it is almost $12 \mathrm{~m}$ for scan pair $\mathcal{P}_{11}$. Due to the significantly larger distance, the similarity between the respective intensity images becomes less and, consequently, the number of feature correspondences decays quickly compared to the other scan pairs. After fine registration, however, the remaining error for scan pair $\mathcal{P}_{11}$ is reduced to the same range as for the other scan pairs, and this behavior also holds for the respective angle errors.

Concerning the involved methods for point quality assessment, the new measure of planarity does not always lead to an improvement after fine registration (Figure 7, center). However, this may be due to the fact the accuracy after fine registration is quite close to the expected measurement accuracy of the scanning device $(12 \mathrm{~mm})$. In this regard, it may be taken into account that the RANSAC-based $\mathrm{EP} n \mathrm{P}$ scheme involves both $3 \mathrm{D}$ and 2D cues, and thus already ensures a relatively reliable coarse registration compared to approaches only focusing on spatial $3 \mathrm{D}$ geometry, where the new measure may show a more significant improvement of the registration results. The main effect of the new method for point quality assessment thus consists of a significant speed-up in coarse registration (Figure 8), while causing additional costs in point quality assessment compared to the other methods (Table 1). The speed-up in coarse registration, in turn, is important since a fast solution corresponds to a reliable esti-

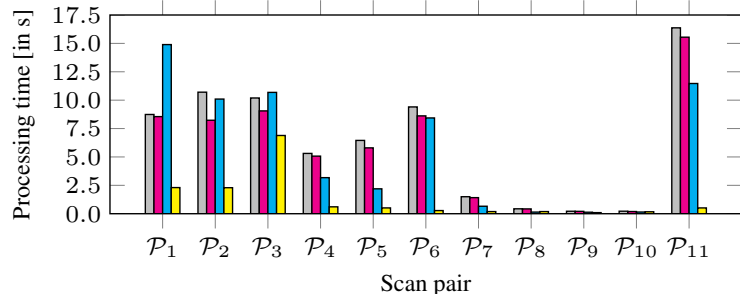

Figure 8. Mean processing times required for the coarse registration of scan pairs $\mathcal{P}_{i}=\left\{\mathcal{S}_{i}, \mathcal{S}_{i+1}\right\}$ when applying no reliability check (gray) and reliability checks w.r.t. intensity (magenta), range reliability (cyan) or planarity (yellow).

mate of the relative orientation between two scans. More specifically, a filtering of feature correspondences based on the proposed measure of planarity represents a consistency check that - like specific modifications of RANSAC (Sattler et al., 2009) results in a reduced set of feature correspondences, where the inlier ratio is significantly increased which, in turn, leads to a faster convergence of the RANSAC algorithm towards a suitable solution. Thereby, the generic consideration of incidence angles up to about $45^{\circ}$ (Section 4) imposes more restrictions than other recent investigations addressing an optimized selection of scan positions (Soudarissanane and Lindenbergh, 2011), where incidence angles up to $70^{\circ}$ are assumed to result in reliable range measurements.

\section{CONCLUSIONS}

In this paper, we have presented an accurate and robust method for a keypoint-based registration of unordered point clouds via projective scan matching. Thereby, robustness is preserved by involving a fully generic consistency check which removes unreliable feature correspondences based on a common measure taking into account the geometric smoothness of object surfaces and the respective incidence angle. As a consequence, the ratio of inlier correspondences is increased which, in turn, leads to a faster convergence of the RANSAC algorithm towards a suitable solution. The results clearly reveal that a highly accurate and robust registration may be achieved in a fully automatic manner without using artificial markers. For future work, it would be desirable to compare different approaches for point cloud registration on a benchmark dataset and to point out pros and cons of these approaches in order to allow end-users to select an appropriate method according to their requirements. Furthermore, it might be advisable to introduce a weighting of feature correspondences which may principally be based on different constraints (Weinmann et al., 2013; Khoshelham et al., 2013).

\section{REFERENCES}

Akca, D., 2003. Full automatic registration of laser scanner point clouds Optical 3-D Measurement Techniques VI, Vol. I, pp. 330-337.

Al-Manasir, K. and Fraser, C. S., 2006. Registration of terrestrial laser scanner data using imagery. The Photogrammetric Record 21(115), pp. 255-268.

Alba, M., Barazzetti, L., Scaioni, M. and Remondino, F., 2011. Automatic registration of multiple laser scans using panoramic RGB and intensity images. The International Archives of the Photogrammetry, Remote Sensing and Spatial Information Sciences, Vol. XXXIII-5/W12, pp. 4954.

Arun, K. S., Huang, T. S. and Blostein, S. D., 1987. Least-squares fitting of two 3-D point sets. IEEE Transactions on Pattern Analysis and Machine Intelligence 9(5), pp. 698-700.

Bae, K.-H. and Lichti, D. D., 2008. A method for automated registration of unorganised point clouds. ISPRS Journal of Photogrammetry and Remote Sensing 63(1), pp. 36-54.

Barber, D., Mills, J. and Smith-Voysey, S., 2008. Geometric validation of a ground-based mobile laser scanning system. ISPRS Journal of Photogrammetry and Remote Sensing 63(1), pp. 128-141. 
Barnea, S. and Filin, S., 2007. Registration of terrestrial laser scans via image based features. The International Archives of the Photogrammetry, Remote Sensing and Spatial Information Sciences, Vol. XXXVI-3/W52, pp. 32-37.

Barnea, S. and Filin, S., 2008. Keypoint based autonomous registration of terrestrial laser point-clouds. ISPRS Journal of Photogrammetry and Remote Sensing 63(1), pp. 19-35.

Besl, P. J. and McKay, N. D., 1992. A method for registration of 3-D shapes. IEEE Transactions on Pattern Analysis and Machine Intelligence 14(2), pp. 239-256.

Boehler, W., Bordas Vicent, M. and Marbs, A., 2003. Investigating laser scanner accuracy. The International Archives of the Photogrammetry, Remote Sensing and Spatial Information Sciences, Vol. XXXIV-5/C15, pp. 1-6.

Boehm, J. and Becker, S., 2007. Automatic marker-free registration of terrestrial laser scans using reflectance features. Optical 3-D Measurement Techniques VIII, pp. 338-344.

Boström, G., Gonçalves, J. G. M. and Sequeira, V., 2008. Controlled 3D data fusion using error-bounds. ISPRS Journal of Photogrammetry and Remote Sensing 63(1), pp. 55-67.

Brenner, C., Dold, C. and Ripperda, N., 2008. Coarse orientation of terrestrial laser scans in urban environments. ISPRS Journal of Photogrammetry and Remote Sensing 63(1), pp. 4-18.

Eggert, D. W., Lorusso, A. and Fisher, R. B., 1997. Estimating 3-D rigid body transformations: a comparison of four major algorithms. Machine Vision and Applications 9(5-6), pp. 272-290.

Fischler, M. A. and Bolles, R. C., 1981. Random sample consensus: a paradigm for model fitting with applications to image analysis and automated cartography. Communications of the ACM 24(6), pp. 381-395.

Franaszek, M., Cheok, G. S. and Witzgall, C., 2009. Fast automatic registration of range images from $3 \mathrm{D}$ imaging systems using sphere targets. Automation in Construction 18(3), pp. 265-274.

Fuchs, S. and May, S., 2008. Calibration and registration for precise surface reconstruction with Time-of-Flight cameras. International Journal of Intelligent Systems Technologies and Applications 5(3/4), pp. 274-284. Gruen, A. and Akca, D., 2005. Least squares 3D surface and curve matching. ISPRS Journal of Photogrammetry and Remote Sensing 59(3), pp. 151-174.

Hartley, R. I. and Zisserman, A., 2008. Multiple view geometry in computer vision. University Press, Cambridge, UK.

Hebert, M. and Krotkov, E., 1992. 3-D measurements from imaging laser radars: how good are they? International Journal of Image and Vision Computing 10(3), pp. 170-178.

Horn, B. K. P., Hilden, H. M. and Negahdaripour, S., 1988. Closed-form solution of absolute orientation using orthonormal matrices. Journal of the Optical Society of America A 5(7), pp. 1127-1135.

Huber, D. F. and Hebert, M., 2003. Fully automatic registration of multiple 3D data sets. Image and Vision Computing 21(7), pp. 637-650.

Kang, Z., Li, J., Zhang, L., Zhao, Q. and Zlatanova, S., 2009. Automatic registration of terrestrial laser scanning point clouds using panoramic reflectance images. Sensors 9(4), pp. 2621-2646.

Khoshelham, K., Dos Santos, D. R. and Vosselman, G., 2013. Generation and weighting of $3 \mathrm{D}$ point correspondences for improved registration of RGB-D data. ISPRS Annals of the Photogrammetry, Remote Sensing and Spatial Information Sciences, Vol. II-5/W2, pp. 127-132.

Lichti, D. D. and Licht, M. G., 2006. Experiences with terrestrial laser scanner modelling and accuracy assessment. The International Archives of the Photogrammetry, Remote Sensing and Spatial Information Sciences, Vol. XXXVI-5, pp. 155-160.

Lichti, D. D., Gordon, S. J. and Tipdecho, T., 2005. Error models and propagation in directly georeferenced terrestrial laser scanner networks. Journal of Surveying Engineering 131(4), pp. 135-142.

Lowe, D. G., 2004. Distinctive image features from scale-invariant keypoints. International Journal of Computer Vision 60(2), pp. 91-110.

Magnusson, M., Lilienthal, A. and Duckett, T., 2007. Scan registration for autonomous mining vehicles using 3D-NDT. Journal of Field Robotics 24(10), pp. 803-827.

Moreno-Noguer, F., Lepetit, V. and Fua, P., 2007. Accurate non-iterative $\mathrm{O}(\mathrm{n})$ solution to the PnP problem. Proceedings of the IEEE International Conference on Computer Vision, pp. 1-8.

Pathak, K., Birk, A., Vaškevičius, N. and Poppinga, J., 2010. Fast registration based on noisy planes with unknown correspondences for 3-D mapping. IEEE Transactions on Robotics 26(3), pp. 424-441.

Pulli, K., Piiroinen, S., Duchamp, T. and Stuetzle, W., 2005. Projective surface matching of colored 3D scans. Proceedings of the International Conference on 3-D Digital Imaging and Modeling, pp. 531-538.
Rabbani, T., Dijkman, S., van den Heuvel, F. and Vosselman, G., 2007. An integrated approach for modelling and global registration of point clouds. ISPRS Journal of Photogrammetry and Remote Sensing 61(6), pp. 355-370.

Sattler, T., Leibe, B. and Kobbelt, L., 2009. SCRAMSAC: Improving RANSAC's efficiency with a spatial consistency filter. Proceedings of the IEEE International Conference on Computer Vision, pp. 2090-2097. Seo, J. K., Sharp, G. C. and Lee, S. W., 2005. Range data registration using photometric features. Proceedings of the IEEE Computer Society Conference on Computer Vision and Pattern Recognition, Vol. 2, pp. 1140-1145.

Soudarissanane, S. and Lindenbergh, R., 2011. Optimizing terrestrial laser scanning measurement set-up. The International Archives of the Photogrammetry, Remote Sensing and Spatial Information Sciences, Vol XXXVIII-5/W12, pp. 127-132.

Soudarissanane, S., Lindenbergh, R., Menenti, M. and Teunissen, P., 2011. Scanning geometry: influencing factor on the quality of terrestrial laser scanning points. ISPRS Journal of Photogrammetry and Remote Sensing 66(4), pp. 389-399.

Stamos, I. and Leordeanu, M., 2003. Automated feature-based range registration of urban scenes of large scale. Proceedings of the IEEE Computer Society Conference on Computer Vision and Pattern Recognition, Vol. 2, pp. 555-561.

Steder, B., Grisetti, G. and Burgard, W., 2010. Robust place recognition for 3D range data based on point features. Proceedings of the IEEE International Conference on Robotics and Automation, pp. 1400-1405.

Steder, B., Grisetti, G., Van Loock, M. and Burgard, W., 2009. Robust online model-based object detection from range images. Proceedings of the IEEE/RSJ International Conference on Intelligent Robots and Systems, pp. 4739-4744.

Steder, B., Rusu, R. B., Konolige, K. and Burgard, W., 2011. Point feature extraction on $3 \mathrm{D}$ range scans taking into account object boundaries. Proceedings of the IEEE International Conference on Robotics and Automation, pp. 2601-2608.

Swadzba, A., Liu, B., Penne, J., Jesorsky, O. and Kompe, R., 2007. A comprehensive system for 3D modeling from range images acquired from a 3D ToF sensor. Proceedings of the International Conference on Computer Vision Systems, pp. 1-10.

Theiler, P. W., Wegner, J. D. and Schindler, K., 2012. Automatic registration of terrestrial laser scanner point clouds using natural planar surfaces. ISPRS Annals of the Photogrammetry, Remote Sensing and Spatial Information Sciences, Vol. I-3, pp. 173-178.

Theiler, P. W., Wegner, J. D. and Schindler, K., 2013. Markerless point cloud registration with keypoint-based 4-points congruent sets. ISPRS Annals of the Photogrammetry, Remote Sensing and Spatial Information Sciences, Vol. II-5/W2, pp. 283-288.

Theiler, P. W., Wegner, J. D. and Schindler, K., 2014. Keypoint-based 4 points congruent sets - Automated marker-less registration of laser scans ISPRS Journal of Photogrammetry and Remote Sensing 96, pp. 149-163. Tuytelaars, T. and Mikolajczyk, K., 2008. Local invariant feature detectors: a survey. Foundations and Trends in Computer Graphics and Vision 3(3), pp. 177-280.

Vosselman, G. and Maas, H.-G., 2010. Airborne and terrestrial lase scanning. Whittles Publishing, Dunbeath, UK

Wang, Z. and Brenner, C., 2008. Point based registration of terrestrial laser data using intensity and geometry features. The International Archives of the Photogrammetry, Remote Sensing and Spatial Information Sciences, Vol. XXXVII-B5, pp. 583-589.

Weinmann, M., 2013. Visual features - From early concepts to modern computer vision. In: Farinella, G. M., Battiato, S. and Cipolla, R. (Eds.), Advanced Topics in Computer Vision. Advances in Computer Vision and Pattern Recognition, Springer, London, UK, pp. 1-34.

Weinmann, M. and Jutzi, B., 2011. Fully automatic image-based registration of unorganized TLS data. The International Archives of the Photogrammetry, Remote Sensing and Spatial Information Sciences, Vol. XXXVIII-5/W12, pp. 55-60.

Weinmann, M., Dittrich, A., Hinz, S. and Jutzi, B., 2013. Automatic feature-based point cloud registration for a moving sensor platform. The International Archives of the Photogrammetry, Remote Sensing and Spatial Information Sciences, Vol. XL-1/W1, pp. 373-378.

Weinmann, M., Weinmann, M., Hinz, S. and Jutzi, B., 2011. Fast and automatic image-based registration of TLS data. ISPRS Journal of Photogrammetry and Remote Sensing 66(6), pp. S62-S70.

Wendt, A., 2007. A concept for feature based data registration by simultaneous consideration of laser scanner data and photogrammetric images ISPRS Journal of Photogrammetry and Remote Sensing 62(2), pp. 122 134.

West, K. F., Webb, B. N., Lersch, J. R., Pothier, S., Triscari, J. M. and Iverson, A. E., 2004. Context-driven automated target detection in 3-D data. Proceedings of SPIE, Vol. 5426, pp. 133-143. 